\title{
Synthesis of Novel Bidentate P-Chiral Diaminophosphine Oxide Preligands: Application to Pd-Catalyzed Asymmetric Allylic Substitution Reactions
}

\author{
Teisuke Harada, Tetsuhiro Nemoto, Long Jin, and Yasumasa HamadA* \\ Graduate School of Pharmaceutical Sciences, Chiba University; 1-33 Yayoi-cho, Inage-ku, Chiba 263-8522, Japan. \\ Received November 27, 2010; accepted December 15, 2010; published online December 15, 2010
}

We developed a novel $(S)$-L-phenylalanine derived-bidentate chiral diaminophosphine oxide (DIAPHOX) preligand $\left(S, S_{\mathrm{P}}\right)-9 \mathrm{~b}$, which was successfully applied to Pd-catalyzed asymmetric allylic alkylation and amination. Using the $\mathrm{Pd}-\left(S, S_{\mathrm{P}}\right)-9 \mathrm{~b}$ catalyst system, asymmetric allylic alkylation and amination proceeded very smoothly, affording the corresponding products in excellent yield with high enantiomeric excess. It is noteworthy that both enantiomers were accessible with high enantiomeric purity using the structurally related DIAPHOX preligands $\left(S, S_{\mathrm{P}}\right)-9 \mathrm{~b}$ and a monodentate chiral diaminophosphine oxide preligand $\left(S, R_{\mathrm{P}}\right)-10 \mathrm{a}$, both of which can be prepared from a single chiral source, $(S)$-L-phenylalanine.

Key word chiral diaminophosphine oxide; asymmetric allylic substitution; palladium; asymmetric catalysis; preligand

Pd-catalyzed asymmetric allylic substitutions have received considerable attention as useful asymmetric carboncarbon bond or carbon-heteroatom bond-forming reactions, where racemic or achiral allylic substrates can be converted into optically active products in the presence of a palladium-chiral ligand complex. Extensive efforts have been directed towards the development of effective chiral ligands for this transformation. ${ }^{1-3)}$ Over the past two decades, we have focused on the development of chiral phosphorus ligands for transition metal-catalyzed asymmetric allylic substitutions. ${ }^{4-7)}$ In 2004, we developed a new class of chiral phosphorus ligands: aspartic acid-derived P-chiral diaminophosphine oxide 1 and its derivatives (DIAPHOXs). These pentavalent phosphorus compounds, preligands, are activated in situ by $N, O$-bis(trimethysilyl)acetamide (BSA) induced $\mathrm{P}(\mathrm{V})$ - $\mathrm{P}(\mathrm{III})$ tautomerization to afford trivalent phosphorus species such as $\mathbf{2}$, which function as actual ligands (Chart 1). These preligands have been successfully applied to Pd- and Ir-catalyzed asymmetric allylic substitution reactions. ${ }^{8-22)}$ Detailed investigations into the reaction mechanism using a Pd-catalyzed asymmetric allylic alkylation of 1,3-diphenylallyl acetate with dimethyl malonate revealed that complex $\mathbf{3}$ [Pd-2 $(1: 2)$ complex] was the active catalyst. ${ }^{9)}$ With this result in hand, we became interested in the development of bidentate P-chiral diaminophosphine oxide preligands with an achiral diphenylphosphine moiety as the second coordinating group. Herein, we report the design and synthesis of novel phenylalanine derived-bidentate P-chiral diaminophosphine oxide preligands that can be applied to Pd-catalyzed asymmetric allylic alkylation and amination.

\section{Results and Discussion}

Our ligand design is outlined in Fig. 1. The original DIAPHOX preligands involve an arylmethylene unit on a nitrogen atom adjacent to the phosphorus atom, which is introduced by reduction of the corresponding benzamide. The commercial availability of 2-diphenylphosphinobenzoic acid led us to introduce a diphenylphosphine moiety into the DIAPHOX framework by reducing the corresponding benzamide prepared from this reagent. Thus, we set up the methyl- ene-tethered P-chiral diaminophosphine oxide-triphenylphosphine hybrids as our target ligands. (S)-Phenylalanine was selected as the starting material to prepare both diastereomers of chiral ligands originating from the chirality of the phosphorus atom. ${ }^{23}$

Our ligand synthesis started with commercially available $N$-Z-(S)-phenylalanine 4 (Chart 2). First, 4 was reacted with aniline using 1-ethyl-3-(3-diethylaminopropyl)-carbodiimide $\cdot$ hydrochloride $(\mathrm{EDC})$ as a coupling reagent to give the corresponding anilide, which was converted into the known primary amine derivative $\mathbf{5}$ through reductive removal of the $Z$ group (94\% yield, 2 steps). Subsequent condensation of $\mathbf{5}$ with 2-diphenylphosphinobenzoic acid using 1 eq of dicyclo-

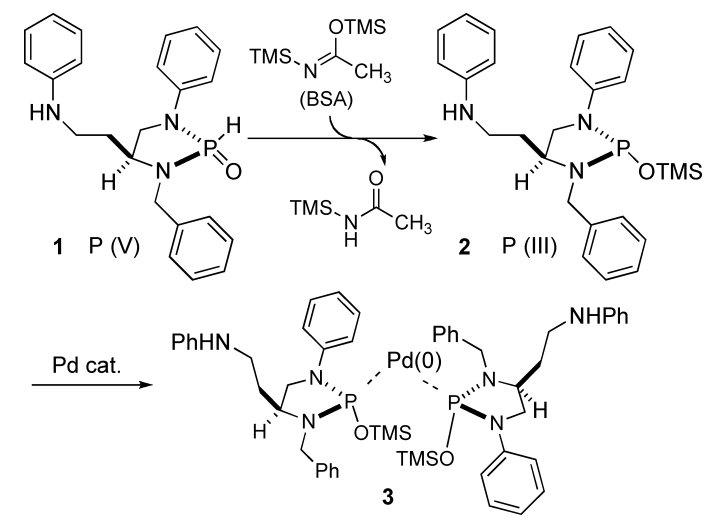

Chart 1. BSA-Induced P(V)-P(III) Tautomerization of DIAPHOXs and the Active Catalyst Structure of the Asymmetric Allylic Substitution Using the Pd-DIAPHOX Catalyst System

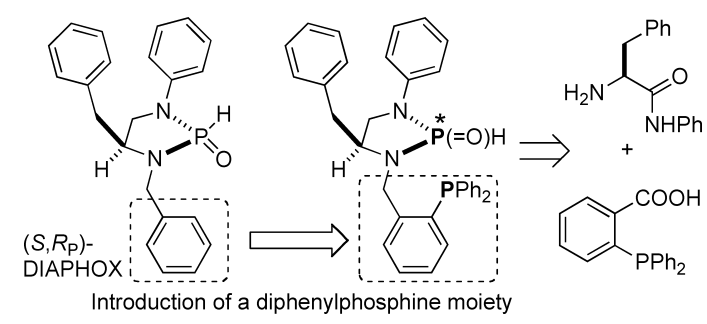

Fig. 1. Design of Bidentate P-Chiral Diaminophosphine Oxide Preligands 

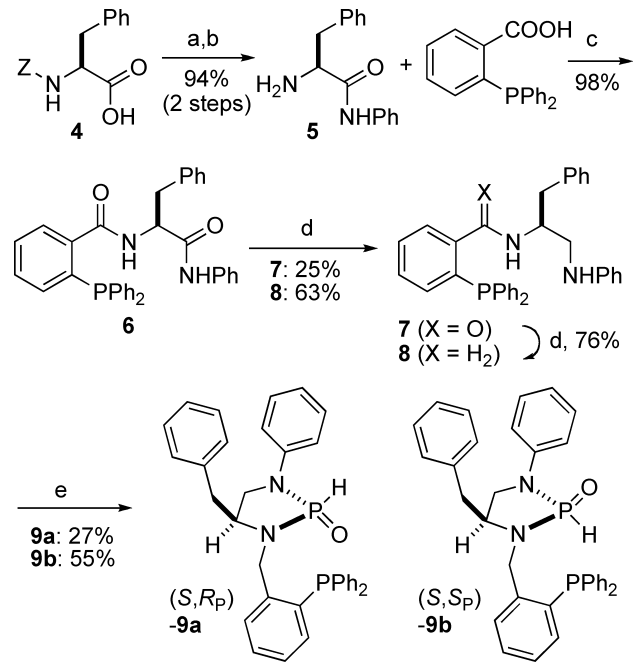

Reagents and conditions: (a) aniline, EDC, DMF, rt. (b) Pd-C, $\mathrm{H}_{2}, \mathrm{DMF}-2$ propanol, rt. (c) DCC, DMAP, $\mathrm{CH}_{2} \mathrm{Cl}_{2}$, rt. (d) $\mathrm{NaBH}_{4}, \mathrm{I}_{2}$, THF, reflux. (e) $\mathrm{PCl}_{3}$, $\mathrm{NEt}_{3}$, toluene, $-78^{\circ} \mathrm{C}$ to $\mathrm{rt}$, then $\mathrm{H}_{2} \mathrm{O}$.

Chart 2. Ligand Synthesis

hexylcarbodiimide (DCC) and $10 \mathrm{~mol} \%$ of $N, N$-dimethylaminopyridine (DMAP) provided 6 in $98 \%$ yield. ${ }^{24}$ Reduction of the amide groups of 6 using in situ-generated $\mathrm{BH}_{3}$ afforded a mixture of the desired diamine 8 (63\% yield) and the monoamine 7 (25\% yield). Recovered 7 was successfully transformed into 8 in $76 \%$ yield by treating with $\mathrm{BH}_{3}$ again (total yield of 8 : $82 \%$ ). Finally, the obtained diamine $\mathbf{8}$ was reacted with phosphorous trichloride in the presence of $\mathrm{NEt}_{3}$. After work-up by the addition of water, the obtained residue was purified by flash column chromatography to give the target compounds $\left(S, R_{\mathrm{P}}\right) \mathbf{- 9} \mathbf{a}$ and $\left(S, S_{\mathrm{P}}\right)-\mathbf{9 b}$ in $27 \%$ yield and $55 \%$ yield, respectively. ${ }^{25)}$

We next used the bidentate P-chiral diaminophosphine oxides $\left(S, R_{\mathrm{P}}\right)-\mathbf{9 a}$ and $\left(S, S_{\mathrm{P}}\right)-\mathbf{9 b}$ as chiral phosphorus ligands for a Pd-catalyzed asymmetric allylic alkylation of 1,3-diphenylallyl acetate 11 with dimethyl malonate (Table 1). We first examined the reaction using the corresponding monodentatetype DIAPHOX $\left(S, R_{\mathrm{P}}\right)-\mathbf{1 0} \mathbf{a}^{16)}$ and $\left(S, S_{\mathrm{P}}\right)-\mathbf{1 0 b ^ { 1 6 ) }}$ as the control experiments. Using $1 \mathrm{~mol} \%$ of $\left[\eta^{3}-\mathrm{C}_{3} \mathrm{H}_{5} \mathrm{PdCl}\right]_{2}$ and $4 \mathrm{~mol} \%$ of $\left(S, R_{\mathrm{P}}\right)-\mathbf{1 0 a}(\mathrm{Pd} /$ ligand $=1 / 2),{ }^{26)}$ the reaction proceeded smoothly at $4{ }^{\circ} \mathrm{C}$ to provide $(S)-12$ in $99 \%$ yield with $95 \%$ ee (entry 1$)$. Using $\left(S, S_{\mathrm{P}}\right) \mathbf{- 1 0 b}$, however, the same reaction proceeded very sluggishly, affording the product with an opposite stereochemistry in only $35 \%$ yield, even after $96 \mathrm{~h}$ (entry 2). In striking contrast, chiral Pd catalysts prepared using $\left(S, R_{\mathrm{P}}\right) \mathbf{- 9} \mathbf{a}$ or $\left(S, S_{\mathrm{P}}\right)-\mathbf{9 b}$ effectively promoted the reaction. In particular, when the reaction was performed using $1 \mathrm{~mol} \%$ of $\left[\eta^{3}-\mathrm{C}_{3} \mathrm{H}_{5} \mathrm{PdCl}\right]_{2}$ and $2 \mathrm{~mol} \%$ of $\left(S, S_{\mathrm{P}}\right)-9 \mathbf{b}(\mathrm{Pd} /$ ligand $=1 / 1)$ at $4{ }^{\circ} \mathrm{C},(R)-12$ was obtained in $99 \%$ yield with $93 \%$ ee (entry $4)$. Conventionally, unnatural $(R)$-D-amino acid-derived DIAPHOX preligands, such as $\left(R, S_{\mathrm{P}}\right)$-10a, were essential to obtain products with an opposite stereochemistry to that obtained with DIAPHOXs bearing $\left(S, R_{\mathrm{P}}\right)$ configurations (entry $5)$. It is noteworthy that both enantiomers were accessible with high enantiomeric purity using the structurally related DIAPHOX preligands derived from a single chiral source, $(S)$-L-phenylalanine. The $\mathrm{Pd}-\left(S, S_{\mathrm{P}}\right)-\mathbf{9 b}$ catalyst system was also applicable to asymmetric allylic amination. When benzylamine and morpholine were utilized as nucleophiles, the
Table 1. Pd-Catalyzed Asymmetric Allylic Alkylation

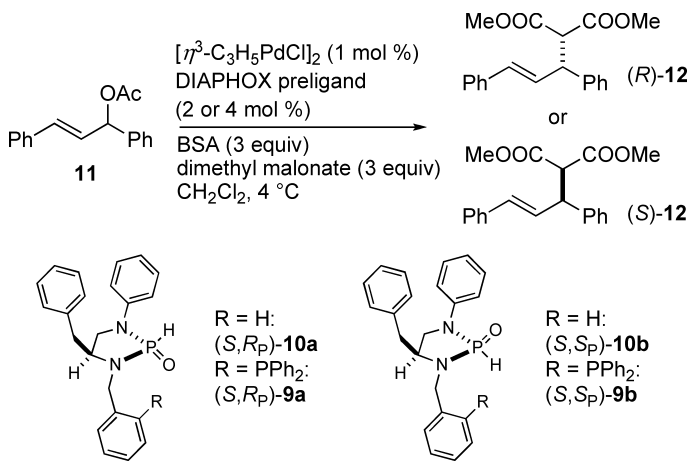

\begin{tabular}{clrlc}
\hline \hline Entry & DIAPHOX & Time & Yield & ee \\
\hline 1 & $\left(S, R_{\mathrm{P}}\right)-\mathbf{1 0 a}(4 \mathrm{~mol} \%)$ & $4 \mathrm{~h}$ & $99 \%$ & $95 \%$ ee $(S)$ \\
2 & $\left(S, S_{\mathrm{P}}\right) \mathbf{- 1 0 b}(4 \mathrm{~mol} \%)$ & $96 \mathrm{~h}$ & $35 \%$ & $89 \%$ ee $(R)$ \\
3 & $\left(S, R_{\mathrm{P}}\right)-\mathbf{9 a}(2 \mathrm{~mol} \%)$ & $0.5 \mathrm{~h}$ & $99 \%$ & $69 \%$ ee $(S)$ \\
4 & $\left(S, S_{\mathrm{P}}\right) \mathbf{- 9 b}(2 \mathrm{~mol} \%)$ & $0.5 \mathrm{~h}$ & $99 \%$ & $93 \%$ ee $(R)$ \\
5 & $\left(R, S_{\mathrm{P}}\right) \mathbf{- 1 0 a}(4 \mathrm{~mol} \%)$ & $4 \mathrm{~h}$ & $99 \%$ & $95 \%$ ee $(R)$ \\
\hline
\end{tabular}
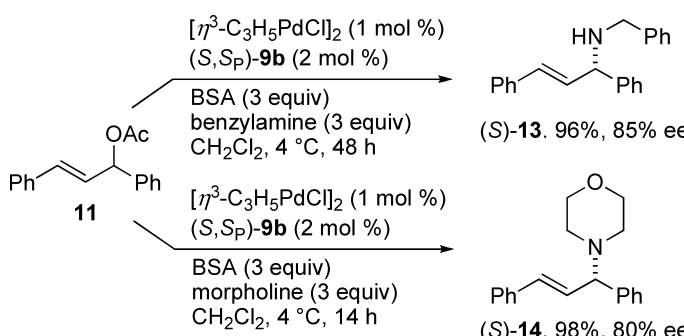

(S)-13. 96\%, 85\% ee

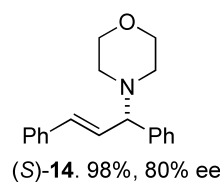

Chart 3. Pd-Catalyzed Asymmetric Allylic Amination

corresponding products $(S)$-13 and $(S)$-14 were obtained in $96 \%$ yield with $85 \%$ ee and in $98 \%$ yield with $80 \%$ ee, respectively (Chart 3).

In conclusion, we developed a novel phenylalanine derived-bidentate chiral diaminophosphine oxide preligand $\left(S, S_{\mathrm{P}}\right)-\mathbf{9 b}$, which was successfully applied to Pd-catalyzed asymmetric allylic alkylation and amination. Both enantiomers were accessible in a highly enantioselective manner using $\left(S, S_{\mathrm{P}}\right)-\mathbf{9 b}$ and $\left(S, R_{\mathrm{P}}\right) \mathbf{- 1 0 a}$, both of which can be prepared from a single chiral source. Detailed mechanistic investigations into the present catalyst system, as well as studies on the application of bidentate-type DIAPHOX preligands to other catalytic asymmetric reactions, are in progress.

\section{Experimental}

General Infrared (IR) spectra were recorded on a JASCO Fourier transform (FT)/IR 230 Fourier transform infrared spectrophotometer, equipped with ATR (Smiths Detection, DuraSample IR II, Canada). NMR spectra were recorded on a JEOL ecp 400 spectrometer, operating at $400 \mathrm{MHz}$ for ${ }^{1} \mathrm{H}-\mathrm{NMR}, 100 \mathrm{MHz}$ for ${ }^{13} \mathrm{C}-\mathrm{NMR}$, and $160 \mathrm{MHz}$ for ${ }^{31} \mathrm{P}-\mathrm{NMR}$. Optical rotations were measured on a JASCO P-1020 polarimeter. Electrospray ionization (ESI) mass spectra were measured on JEOL AccuTOF LC-plus JMST100LP. The enantiomeric excess was determined by HPLC analysis. HPLC was performed on JASCO HPLC systems consisting of the following: pump, PU-980; detector, UV-970, measured at $254 \mathrm{~nm}$. Reactions were carried out in dry solvent. Other reagents were purified by the usual methods.

Preparation of Bidentate P-Chiral Diaminophosphine Oxide $\left(S, R_{\mathrm{P}}\right)$ 9a and $\left(\boldsymbol{S}, S_{\mathrm{P}}\right)-9 \mathrm{~b}$. Compound 6 To a stirred solution of $\mathbf{5}(200 \mathrm{mg}$, $0.832 \mathrm{mmol}$ ), which was prepared using the reported method ( $94 \%$ yield, 2 steps from 4), ${ }^{16)}$ 2-diphenylphosphinobenzoic acid $(280.4 \mathrm{mg}, 0.915 \mathrm{mmol})$ 
and DMAP (111.8 $\mathrm{mg}, 0.915 \mathrm{mmol})$ in $\mathrm{CH}_{2} \mathrm{Cl}_{2}(4.1 \mathrm{ml})$ at room temperature was added DCC (206.3 mg, $1.00 \mathrm{mmol})$. The resulting mixture was kept stirring at the same temperature. After $24 \mathrm{~h}$, the mixture was filtered through a short pad of celite, and the filtrate was evaporated under reduced pressure. The crude residue was purified by flash column chromatography $\left(\mathrm{SiO}_{2}\right.$, hexane/AcOEt $=10 / 1)$ to give $6(430.9 \mathrm{mg}, 98 \%$ yield $)$ as white solids. IR (ATR) $v$ 3264, 3058, 1678, 1618, 1584, 1530, 1496, 1444, 1434, 1389, $1299,1251,738,692 \mathrm{~cm}^{-1} ;{ }^{1} \mathrm{H}-\mathrm{NMR}\left(\mathrm{CDCl}_{3}\right) \delta: 2.91(\mathrm{dd}, J=7.6,14.4 \mathrm{~Hz}$, $1 \mathrm{H}), 3.20(\mathrm{dd}, J=6.8,14.4 \mathrm{~Hz}, 1 \mathrm{H}), 4.84(\mathrm{ddd}, J=6.8,7.2,7.6 \mathrm{~Hz}, 1 \mathrm{H}), 6.33$ $(\mathrm{d}, J=7.2 \mathrm{~Hz}, 1 \mathrm{H}), 6.97-7.01(\mathrm{~m}, 1 \mathrm{H}), 7.06-7.11(\mathrm{~m}, 2 \mathrm{H}), 7.21-7.38(\mathrm{~m}$, $18 \mathrm{H}), 7.40-7.44(\mathrm{~m}, 1 \mathrm{H}), 7.48-7.50(\mathrm{~m}, 2 \mathrm{H}), 8.44(\mathrm{~s}, 1 \mathrm{H}) ;{ }^{13} \mathrm{C}-\mathrm{NMR}$ $\left(\mathrm{CDCl}_{3}\right) \delta: 37.5,55.9,120.2(2 \mathrm{C}), 124.3,127.0,127.8(\mathrm{~d}, J=5.8 \mathrm{~Hz}), 128.7$ $(\mathrm{d}, J=7.2 \mathrm{~Hz})(2 \mathrm{C}), 128.7(2 \mathrm{C}), 128.8(2 \mathrm{C}), 128.9(\mathrm{~d}, J=7.3 \mathrm{~Hz})(2 \mathrm{C}), 129.2$ (2C), $128.8-129.2(\mathrm{~m}, 3 \mathrm{C}), 130.6,133.6(\mathrm{~d}, J=13.0 \mathrm{~Hz})(2 \mathrm{C}), 133,8(\mathrm{~d}$, $J=13.4 \mathrm{~Hz}$ ) (2C), 134.3, 135.5-138.4 (m, 3C), 136.5, 137.6, 140.5, 168.6, 169.4; ${ }^{31} \mathrm{P}-\mathrm{NMR}\left(\mathrm{CDCl}_{3}\right) \delta$ : -10.7 ; ESI-high resolution (HR)-MS. Calcd for $\mathrm{C}_{34} \mathrm{H}_{29} \mathrm{~N}_{2} \mathrm{NaO}_{2} \mathrm{P}\left(\mathrm{M}+\mathrm{Na}^{+}\right)$: 551.1864. Found: 551.1858; $[\alpha]_{\mathrm{D}}^{23}-48.0$ (c=2.41, $\mathrm{CHCl}_{3}$ ).

Compound 8 To a stirred suspension of $6(427.3 \mathrm{mg}, 0.81 \mathrm{mmol})$ and $\mathrm{NaBH}_{4}(184.7 \mathrm{mg}, 4.86 \mathrm{mmol})$ in tetrahydrofuran (THF) $(4.1 \mathrm{ml})$ was added a THF solution of $\mathrm{I}_{2}(308.4 \mathrm{mg}, 2.43 \mathrm{mmol}$ in $4 \mathrm{ml}$ of $\mathrm{THF})$ at $0^{\circ} \mathrm{C}$ over $3 \mathrm{~min}$. The reaction mixture was refluxed for $48 \mathrm{~h}$, cooled to $0^{\circ} \mathrm{C}$, and then $1 \mathrm{~N} \mathrm{HCl}$ was added to the reaction mixture. After refluxing for $2 \mathrm{~h}$, the reaction mixture was neutralized using $1 \mathrm{~N} \mathrm{NaOH}$. The organic layer was separated and aqueous layer was extracted with AcOEt. The combined organic layers were washed with water, brine, and dried over $\mathrm{Na}_{2} \mathrm{SO}_{4}$. After concentration under reduced pressure, the residue was purified by flash column chromatography $\left(\mathrm{SiO}_{2}\right.$, hexane/AcOEt $=10 / 1$ to $\left.2 / 1\right)$ to give diamine 8 (255.0 mg, 63\% yield) and monoamine 7 (102.9 mg, 25\% yield). Compound 7 could be transformed into 8 in $76 \%$ yield by treating with the same reaction conditions again (total yield of 8 : 82\%). IR (ATR) $v 3368,3324,3052$, 3024, 2921, 2851, 1602, 1505, 1477, 744, $694 \mathrm{~cm}^{-1} ;{ }^{1} \mathrm{H}-\mathrm{NMR}\left(\mathrm{CDCl}_{3}\right) \delta$ : $2.64-2.74(\mathrm{~m}, 2 \mathrm{H}), 2.90(\mathrm{dd}, J=5.6,11.6 \mathrm{~Hz}, 1 \mathrm{H}), 3.03-3.08(\mathrm{~m}, 1 \mathrm{H})$, $3.10(\mathrm{dd}, J=7.6,11.6 \mathrm{~Hz}, 1 \mathrm{H}), 3.93(\mathrm{dd}, J=1.6,12.8 \mathrm{~Hz}, 1 \mathrm{H}), 4.00$ (d, $J=12.8 \mathrm{~Hz}, 1 \mathrm{H}), 4.30-4.70($ broad peak $(\mathrm{NH}), 2 \mathrm{H}), 6.54-6.56(\mathrm{~m}, 2 \mathrm{H})$, $6.64-6.68(\mathrm{~m}, 1 \mathrm{H}), 6.88-6.91(\mathrm{~m}, 1 \mathrm{H}), 7.06-7.38(\mathrm{~m}, 20 \mathrm{H}) ;{ }^{13} \mathrm{C}-\mathrm{NMR}$ $\left(\mathrm{CDCl}_{3}\right) \delta: 39.2,45.2,49.8(\mathrm{~d}, J=19.8 \mathrm{~Hz}), 57.9,113.0(2 \mathrm{C}), 116.9,126.2$, $127.4,128.4-129.3(\mathrm{~m}, 14 \mathrm{C}), 133.7(\mathrm{~d}, J=9.8 \mathrm{~Hz})(2 \mathrm{C}), 133.9(\mathrm{~d}$, $J=9.8 \mathrm{~Hz})(2 \mathrm{C}), 134.0,135.9(\mathrm{~d}, J=14.0 \mathrm{~Hz}), 136.8(\mathrm{~d}, J=9.9 \mathrm{~Hz}), 136.9$ (d, $J=9.9 \mathrm{~Hz}), 138.8,144.3(\mathrm{~d}, J=23.8 \mathrm{~Hz}), 148.8 ;{ }^{31} \mathrm{P}-\mathrm{NMR}\left(\mathrm{CDCl}_{3}\right) \quad \delta$ : -15.4; ESI-HR-MS. Calcd for $\mathrm{C}_{34} \mathrm{H}_{34} \mathrm{~N}_{2} \mathrm{P}\left(\mathrm{M}+\mathrm{H}^{+}\right)$: 501.2460. Found: $501.2453 ;[\alpha]_{\mathrm{D}}^{24}+3.55\left(c=0.81, \mathrm{CHCl}_{3}\right)$.

Compound $\left(S, R_{\mathrm{P}}\right)-9 \mathrm{a}$ and $\left(\boldsymbol{S}, \boldsymbol{S}_{\mathrm{P}}\right)-9 \mathrm{~b}$ To a stirred solution of $\mathbf{8}$ $(721.1 \mathrm{mg}, 1.44 \mathrm{mmol})$ and triethylamine $(0.783 \mathrm{ml}, 5.62 \mathrm{mmol})$ in toluene $(14.4 \mathrm{ml})$ at $-78^{\circ} \mathrm{C}$ was added phosphorous trichloride $(0.138 \mathrm{ml}$, $1.58 \mathrm{mmol}$ ) over $3 \mathrm{~min}$. The reaction temperature was gradually warmed up to room temperature, and then the mixture was kept stirring for $12 \mathrm{~h}$. After diluting with AcOEt, the reaction was quenched with the addition of water. The obtained mixture was washed with water, brine, and then dried over $\mathrm{Na}_{2} \mathrm{SO}_{4}$. After concentration in vacuo, the obtained residue was purified by flash column chromatography $\left(\mathrm{SiO}_{2}\right.$, hexane/AcOEt $=4 / 1$ to $\left.1 / 1\right)$ to give $\left(S, R_{\mathrm{P}}\right)-\mathbf{9 a}(213.1 \mathrm{mg}, 27 \%$ yield $)$ and $\left(S, S_{\mathrm{P}}\right)-9 \mathbf{b}(431.8 \mathrm{mg}, 55 \%$ yield $)$ as white solids. $\left(S, R_{\mathrm{P}}\right)-9 \mathrm{a}$ : IR (ATR) $v$ 3068, 2926, 1600, 1500, 1434, 1302, 1232, 1204, 1166, 1109, 1029, 963, 744, $695 \mathrm{~cm}^{-1} ;{ }^{1} \mathrm{H}-\mathrm{NMR}\left(\mathrm{CDCl}_{3}\right) \delta$ : $2.44(\mathrm{dd}, J=10.0,13.2 \mathrm{~Hz}, 1 \mathrm{H}), 3.13(\mathrm{dd}, J=3.6,13.2 \mathrm{~Hz}, 1 \mathrm{H}), 3.24$ (ddd, $\left.J=4.4 \mathrm{~Hz}\left(\mathrm{CH}_{2}-\mathrm{NPh}-\underline{\mathrm{P}}(=\mathrm{O}) \mathrm{H}\right), 9.6,9.6 \mathrm{~Hz}, 1 \mathrm{H}\right), 3.44$ (ddd, $J=6.0 \mathrm{~Hz}$ $\left.\left(\mathrm{CH}_{2}-\mathrm{NPh}-\underline{\mathrm{P}}(=\mathrm{O}) \mathrm{H}\right), 6.0,9.6 \mathrm{~Hz}, 1 \mathrm{H}\right), 3.68-3.78(\mathrm{~m}, 1 \mathrm{H}), 4.38(\mathrm{dd}$, $\left.J=9.6 \mathrm{~Hz} \quad\left(\mathrm{H}(\mathrm{O}=) \underline{\mathrm{P}}-\mathrm{NCH}_{2} \mathrm{Ar}\right), \quad 15.6 \mathrm{~Hz}, \quad 1 \mathrm{H}\right), \quad 4.90 \quad(\mathrm{ddd}, \quad J=5.2 \mathrm{~Hz}$ $\left.\left(\mathrm{Ph}_{2} \underline{\mathrm{P}}-\mathrm{C}-\mathrm{C}-\mathrm{CH}_{2} \mathrm{~N}\right), 10.8 \mathrm{~Hz}\left(\mathrm{H}(\mathrm{O}=) \underline{\mathrm{P}}-\mathrm{NC}_{2} \mathrm{Ar}\right), 15.6 \mathrm{~Hz}, 1 \mathrm{H}\right), 6.87-7.08$ $(\mathrm{m}, 7 \mathrm{H}), 7.20-7.52(\mathrm{~m}, 16 \mathrm{H}), 7.58(\mathrm{~d}, J=629.2 \mathrm{~Hz}(\underline{\mathrm{P}}(=\mathrm{O}) \underline{\mathrm{H}}), 1 \mathrm{H}), 7.82$ $7.85(\mathrm{~m}, 1 \mathrm{H}) ;{ }^{13} \mathrm{C}-\mathrm{NMR}\left(\mathrm{CDCl}_{3}\right) \delta: 39.4,43.8(\mathrm{dd}, J=6.5,27.5 \mathrm{~Hz}), 47.8(\mathrm{~d}$, $J=9.5 \mathrm{~Hz}), 56.1(\mathrm{~d}, J=6.9 \mathrm{~Hz}), 115.8(\mathrm{~d}, J=5.3 \mathrm{~Hz})(2 \mathrm{C}), 121.6,126.8$, $128.4-132.1(\mathrm{~m}, 15 \mathrm{C}), 133.3,133.7$ (d, $J=19.5 \mathrm{~Hz})(2 \mathrm{C}), 134.1$ (d, $J=19.5 \mathrm{~Hz})(2 \mathrm{C}), 135.8(\mathrm{~d}, J=14.1 \mathrm{~Hz}), 136.0(\mathrm{~d}, J=9.2 \mathrm{~Hz}), 136.8,141.2$ $(\mathrm{dd}, J=3.4,23.6 \mathrm{~Hz}), 141.6(\mathrm{~d}, J=7.3 \mathrm{~Hz}) ;{ }^{31} \mathrm{P}-\mathrm{NMR}\left(\mathrm{CDCl}_{3}\right) \delta:-16.3(\mathrm{~d}$, $J=10.2 \mathrm{~Hz}$ ), 12.4 (d, $J=10.2 \mathrm{~Hz}) ;{ }^{31} \mathrm{P}-\mathrm{NMR}\left(\mathrm{CDCl}_{3}\right) \delta:-15.3,11.3$; ESIHR-MS. Calcd for $\mathrm{C}_{34} \mathrm{H}_{32} \mathrm{~N}_{2} \mathrm{NaOP}_{2}\left(\mathrm{M}+\mathrm{Na}^{+}\right)$: 569.1888. Found: 569.1900; $[\alpha]_{\mathrm{D}}^{24}-68.0\left(c=0.41, \mathrm{CHCl}_{3}\right) .\left(S, S_{\mathrm{P}}\right)-9 \mathbf{b}$ : IR (ATR) $v 3051,2926,1600$, $1497,1473,1434,1301,1228,1166,965,742,697 \mathrm{~cm}^{-1}$; ${ }^{1} \mathrm{H}-\mathrm{NMR}\left(\mathrm{CDCl}_{3}\right)$ $\delta: 2.72-2.42(\mathrm{~m}, 1 \mathrm{H}), 2.73(\mathrm{dd}, J=10.8,13.2 \mathrm{~Hz}, 1 \mathrm{H}), 3.03-3.09(\mathrm{~m}, 1 \mathrm{H})$, $3.24-3.33(\mathrm{~m}, 1 \mathrm{H}), 3.34(\mathrm{dd}, J=3.6,13.2 \mathrm{~Hz}, 1 \mathrm{H}), 4.55(\mathrm{dd}, J=8.8 \mathrm{~Hz}$ $\left.\left(\mathrm{H}(\mathrm{O}=) \underline{\mathrm{P}}-\mathrm{NC} \underline{\mathrm{H}}_{2} \mathrm{Ar}\right), 14.4 \mathrm{~Hz}, 1 \mathrm{H}\right), 4.79\left(\mathrm{dd}, J=6.8 \mathrm{~Hz}\left(\mathrm{H}(\mathrm{O}=) \underline{\mathrm{P}}-\mathrm{NC} \underline{\mathrm{H}}_{2} \mathrm{Ar}\right)\right.$, $14.4 \mathrm{~Hz}, 1 \mathrm{H}), 6.94-7.06(\mathrm{~m}, 6 \mathrm{H}), 7.14-7.33(\mathrm{~m}, 16 \mathrm{H}), 7.40-7.44(\mathrm{~m}$, $1 \mathrm{H}), 7.46(\mathrm{~d}, J=628.8 \mathrm{~Hz}(\underline{\mathrm{P}}(=\mathrm{O}) \underline{\mathrm{H}}), 1 \mathrm{H}), 7.54-7.57(\mathrm{~m}, 1 \mathrm{H}) ;{ }^{13} \mathrm{C}-\mathrm{NMR}$
$\left(\mathrm{CDCl}_{3}\right) \delta: 38.4,45.6(\mathrm{dd}, J=7.0,20.5 \mathrm{~Hz}), 47.8(\mathrm{~d}, J=9.2 \mathrm{~Hz}), 56.1(\mathrm{~d}$, $J=6.5 \mathrm{~Hz}), 115.7(\mathrm{~d}, J=5.3 \mathrm{~Hz})(2 \mathrm{C}), 121.6,126.6,128.4-129.3(\mathrm{~m}, 9 \mathrm{C})$, $129.2(\mathrm{~d}, J=5.4 \mathrm{~Hz})(2 \mathrm{C}), 129.2(\mathrm{~d}, J=5.4 \mathrm{~Hz})(2 \mathrm{C}), 130.1(\mathrm{~d}, J=5.4 \mathrm{~Hz})$, $133.5(\mathrm{~d}, J=19.5 \mathrm{~Hz})(2 \mathrm{C}), 133.5(\mathrm{~d}, J=19.5 \mathrm{~Hz})(2 \mathrm{C}), 135.1,136.0(\mathrm{~d}$, $J=8.8 \mathrm{~Hz}), 136.5(\mathrm{~d}, J=3.1 \mathrm{~Hz}), 136.6(\mathrm{~d}, J=9.1 \mathrm{~Hz}), 137.9,141.2$ (dd, $J=6.1,25.2 \mathrm{~Hz}), 141.9(\mathrm{~d}, J=8.1 \mathrm{~Hz}) ;{ }^{31} \mathrm{P}-\mathrm{NMR}\left(\mathrm{CDCl}_{3}\right) \delta:-16.3(\mathrm{~d}$, $J=10.2 \mathrm{~Hz}$ ), 12.4 (d, $J=10.2 \mathrm{~Hz}$ ); ESI-HR-MS. Calcd for $\mathrm{C}_{34} \mathrm{H}_{32} \mathrm{~N}_{2} \mathrm{NaOP}_{2}$ $\left(\mathrm{M}+\mathrm{Na}^{+}\right): 569.1888$. Found: $569.1892 ;[\alpha]_{\mathrm{D}}^{23}+38.4\left(c=0.58, \mathrm{CHCl}_{3}\right)$.

General Procedure for the Pd-Catalyzed Asymmetric Allylic Alkylation Using $\left(\boldsymbol{S}, \boldsymbol{S}_{\mathbf{P}}\right)-\mathbf{9 b}$ To a stirred solution of $\left[\eta^{3}-\mathrm{C}_{3} \mathrm{H}_{5} \mathrm{PdCl}\right]_{2}(1.1 \mathrm{mg}$, $0.003 \mathrm{mmol}),\left(S, S_{\mathrm{P}}\right)-\mathbf{9 b}(3.3 \mathrm{mg}, 0.006 \mathrm{mmol})$ in $\mathrm{CH}_{2} \mathrm{Cl}_{2}(1 \mathrm{ml})$ at room temperature was added BSA $(222 \mathrm{ml}, 0.90 \mathrm{mmol})$. After being stirred for $10 \mathrm{~min}$ at the same temperature, a $\mathrm{CH}_{2} \mathrm{Cl}_{2}$ solution of $11(76.0 \mathrm{mg}, 0.30 \mathrm{mmol}$ in $0.5 \mathrm{ml}$ of $\left.\mathrm{CH}_{2} \mathrm{Cl}_{2}\right)$ and dimethyl malonate $(103 \mathrm{ml}, 0.90 \mathrm{mmol})$ were added successively to the reaction at $4{ }^{\circ} \mathrm{C}$. After $30 \mathrm{~min}$, the reaction was concentrated under reduced pressure. The obtained residue was purified by flash column chromatography $\left(\mathrm{SiO}_{2}\right.$, hexane/AcOEt, 30/1) to give $(R)-\mathbf{1 2}^{9)}$ $(96.3 \mathrm{mg}, 99 \%$ yield, $93 \%$ ee) as colorless oil. The enantiomeric excess was determined by HPLC analysis (DAICEL CHIRALPAK AD-H, 2propanol/hexane $8 / 92$, flow rate $0.5 \mathrm{ml} / \mathrm{min}, t_{\mathrm{R}} 22.9 \mathrm{~min}[(R)$-isomer] and $31.8 \mathrm{~min}[(S)$-isomer], detection at $254 \mathrm{~nm})$.

Compound $(\boldsymbol{S}) \mathbf{- 1 3}^{11)}$ The enantiomeric excess was determined by HPLC analysis (DAICEL CHIRALCEL OJ-H, 2-propanol/hexane 15/85, flow rate $0.4 \mathrm{ml} / \mathrm{min}, t_{\mathrm{R}} 27.1 \mathrm{~min}[(S)$-isomer] and $35.3 \mathrm{~min}[(R)$-isomer], detection at $254 \mathrm{~nm})$.

Compound $(S)-14^{11)}$ The enantiomeric excess was determined by HPLC analysis (DAICEL CHIRALCEL OJ-H, 2-propanol/hexane 5/95, flow rate $0.5 \mathrm{ml} / \mathrm{min}, t_{\mathrm{R}} 20.5 \mathrm{~min}[(S)$-isomer] and $29.1 \mathrm{~min}$ [ $(R)$-isomer], detection at $254 \mathrm{~nm})$.

Acknowledgments This work was supported in part by a Grant-in-Aid for Encouragement of Young Scientists (B) from the Ministry of Education, Culture, Sports, Science and Technology of Japan.

\section{References and Notes}

1) For a review of transition metal-catalyzed asymmetric allylic substitutions, see: Trost B. M., Van Vranken D. L., Chem. Rev., 96, 395-422 (1996).

2) For a review of transition metal-catalyzed asymmetric allylic substitutions, see: Trost B. M., Crawley M. L., Chem. Rev., 103, 2921-2943 (2003).

3) For a review of transition metal-catalyzed asymmetric allylic substitutions, see: Lu Z., Ma S., Angew. Chem. Int. Ed., 47, 258-297 (2008).

4) Hamada Y., Seto N., Ohmori H., Hatano K., Tetrahedron Lett., 37, 7565 -7568 (1996).

5) Hamada Y., Seto N., Takayanagi Y., Nakano T., Hara O., Tetrahedron Lett., 40, 7791-7794 (1999).

6) Hamada Y., Sakaguchi K., Hatano K., Hara O., Tetrahedron Lett., 42, 1297-1299 (2001).

7) Hara O., Koshizawa T., Makino K., Kunimune I., Namiki A., Hamada Y., Tetrahedron, 63, 6170-6181 (2007).

8) Nemoto T., Matsumoto T., Masuda T., Hitomi T., Hatano K., Hamada Y., J. Am. Chem. Soc., 126, 3690-3691 (2004).

9) Nemoto T., Masuda T., Matsumoto T., Hamada Y., J. Org. Chem., 70, $7172-7178$ (2005)

10) Nemoto T., Fukuda T., Matsumoto T., Hitomi T., Hamada Y., Adv. Synth. Catal., 347, 1504-1506 (2005).

11) Nemoto T., Masuda T., Akimoto Y., Fukuyama T., Hamada Y., Org. Lett., 7, 4447-4450 (2005).

12) Nemoto T., Jin L., Nakamura H., Hamada Y., Tetrahedron Lett., 47, 6577 -6581 (2006)

13) Nemoto T., Sakamoto T., Matsumoto T., Hamada Y., Tetrahedron Lett., 47, 8737-8740 (2006).

14) Nemoto T., Fukuyama T., Yamamoto E., Tamura S., Fukuda T., Matsumoto T., Akimoto Y., Hamada Y., Org. Lett., 9, 927-930 (2007).

15) Nemoto T., Sakamoto T., Fukuyama T., Hamada Y., Tetrahedron Lett., 48, 4977-4981 (2007).

16) Nemoto T., Harada T., Matsumoto T., Hamada Y., Tetrahedron Lett., 48, $6304-6307$ (2007).

17) Jin L., Nemoto T., Nakamura H., Hamada Y., Tetrahedron: Asymmetry, 19, 1106-1113 (2008).

18) Nemoto T., Tamura S., Sakamoto T., Hamada Y., Tetrahedron: Asymmetry, 19, 1751-1759 (2008).

19) Nemoto T., Kanematsu M., Tamura S., Hamada Y., Adv. Synth. Catal., 
351, 1773-1778 (2009).

20) Nemoto T., Yamamoto E., Franzén R., Fukuyama T., Wu R., Fukamachi T., Kobayashi H., Hamada Y., Org. Lett., 12, 872-875 (2010).

21) For a review, see: Nemoto T., Hamada Y., Chem. Rec., 7, 150-158 (2007).

22) For a review, see: Nemoto T., Chem. Pharm. Bull., 56, 1213-1228 (2008).

23) Aspartic acid-derived P-chiral DIAPHOX preligands with $\left(S, S_{\mathrm{P}}\right)$ configurations are not accessible because of the characteristic reaction pathway in the stage of introduction of the phosphine oxide moiety. See ref. 9 for details.

24) Breit B., Laungani A. C., Tetrahedron: Asymmetry, 14, 3823-3826 (2003).

25) The absolute configuration of the phosphorus atom of $9 \mathbf{a}$ and $9 \mathbf{b}$ was predicted by comparing the measured optical rotation with that of $\left(S, R_{\mathrm{P}}\right)-(-)-\mathbf{1 0 a}$ and $\left(S, S_{\mathrm{P}}\right)-(+)-\mathbf{1 0 b}$.

26) The Pd-ligand ratio $(\mathrm{Pd} / \mathbf{1 0 a}$ or $\mathrm{Pd} / \mathbf{1 0 b}=1 / 2)$ was set based on the previous examinations using DIAPHOX 1. See ref. 9 for details. 